\title{
Revision of Hip Resurfacing Arthroplasty with a Bone-conserving Short-stem Implant
}

\author{
Florian Schmidutz*, Lorenz Wanke-Jellinek*, Volkmar Jansson*, \\ Andreas Fottner*, and Farhad Mazoochian*
}

As this case report demonstrates, revision of hip resurfacing arthroplasty can be performed not only with a conventional hip implant, but also with a bone-conserving short-stem hip implant, providing a long-term solution to younger patients with a high risk of further revisions.

\section{Introduction}

The good clinical results in hip arthroplasty have led to an increasing number of joint replacements in younger patients. Regardless, it is well known that this patient group faces an increased risk of early implant failure, [1] which is probably related to their higher activity level. Revision surgeries often go along with loss of bone substance, [2] resulting in more difficult procedures and an impaired functional outcome. [3] To facilitate potential revision surgeries, bone-preserving implants, such as hip resurfacing arthroplasty (HRA) and short-stem arthroplasty (SHA) implants, have been developed and recently have gained increasing popularity.

However, data are lacking on how much bone stock is conserved - and whether revision procedures are actually facilitated by the use of bone-preserving implants. So far, only a few studies have reported on the revision of failed HRA implants, and all revisions have exclusively been performed by the use of a conventional stem. $[4,5]$

In this report, we describe a woman with early failure of HRA. Revision was performed with a bone-conserving short-stem hip implant, which minimizes the bone loss on the femoral side in order to facilitate potential revision surgery.

\section{Case Presentation}

A 56-year-old Caucasian woman presented to the outpatient clinic of our department with osteoarthritis of the left hip of approximately 6 years. As con-

servative treatment had failed, she requested hip replacement arthroplasty. Due to her comparatively younger age and activity level, HRA (Cormet, Corin Group, Cirencester, UK) was performed.

Her postoperative course was unremarkable, and the final radiologic assessment showed an implant position with a cup inclination of $50^{\circ}$ and a stemshaft angle of $132^{\circ}$. Our patient fully recovered, and the follow-up investigation revealed a stable implant. The patient did not report any pain or problems related to the implant.

Three years later, the patient presented outside the normal follow-up with severe pain in the replaced hip joint. She reported a falling incident that had occurred 2 months earlier, followed by an increasing pain over the subsequent weeks.

Clinical and radiologic evaluation revealed a failure of the acetabular component, which was already dislocated, and additionally showed a narrowing of the femoral neck (Figure 1). For those reasons, the patient underwent revision surgery.

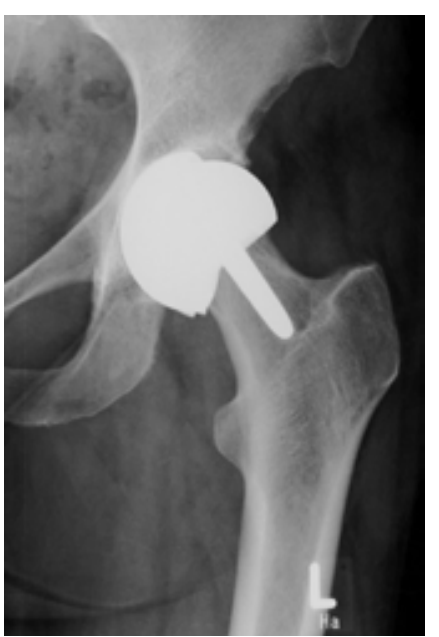

Figure 1. Early failure of hip resurfacing arthroplasty three years after implantation. 


\section{Management}

Intraoperatively, a massive metallosis of the periprosthetic tissue was found, and the femoral and acetabular components were already damaged. Therefore, removal of the whole implant became necessary.

As the femoral bone was found to be intact, osteotomy could be performed directly below the femoral component. By doing this, preservation of the femoral neck was possible, which allowed a revision with a metaphyseal-anchored short-stem hip implant (Metha, B. Braun AesculapOrthopedics, Tuttlingen, Germany).

Postoperatively, our patient recovered well and was subsequently referred to a rehabilitation facility. Mobilization was performed by default with half body weight until soft tissue healing was accomplished ( 2 weeks), followed by a rapid and painadapted increase to full weight-bearing. The followup visits at $1,3,6,12$, and 24 months postoperatively were normal. The radiographs at the 2-year follow-up showed a stable implant position (Figures 2a-b).

Clinical function 2 years after revision was good, with a Harris Hip Score of 86, a University

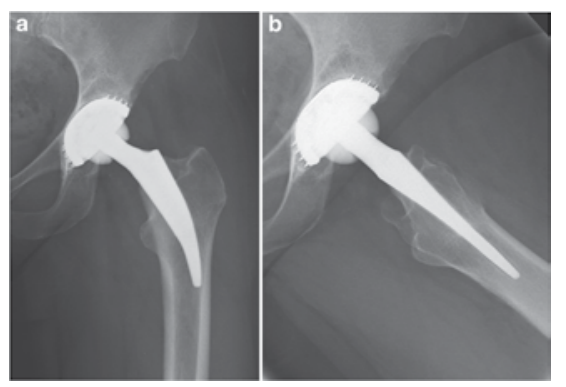

Figures 2 a-b. Short-stem hip implant two years after revision surgery in (a) anterior-posterior and (b) lateral views. of California Los Angeles(UCLA) score of six, and a Western Ontario and McMaster Universities Arthritis Index (WOMAC) score of 12.6, with 3.8 in the category "pain," 1.7 in the category "stiffness," and 7.1 in the category "function."

\section{Discussion}

Preservation of bone stock in younger patients requiring hip replacement is important because those patients will most likely experience at least one implant revision during their remaining lifetime. [1] Our patient was provided with HRA, as the implant design had shown good clinical function and dislocation rates as well as high sports activity levels. [6,7] Furthermore, several studies have demonstrated a satisfying mid-term and long-term outcome. [6] However, it has recently become apparent that HRA also compromises the risk of early failure in certain patients, especially in women with small implants, as seen in our patient. [8]

Although we are not able to state what finally caused the early implant failure in our patient's case, HRA preserved femoral bone stock and thereby facilitated revision surgery. This is of major importance as, beside damage of the soft tissue, bone loss represents one of the main reasons leading to an impaired function after revision surgery. [3] Because many patients with a failed HRA are less than 60 years of age, $[4,5]$ it is necessary to devise a long-term strategy.

Up until now, published data have described revision of HRA with a conventional hip stem. [4,5,9] Moreover, SHA has so far only been used for primary hip replacement. [10-12] Sanguesa-Nebot et al reported the case of a patient with a broken cementless conventional stem that was revised with SHA. [13] As the tip of the implant was broken and stuck in the distal femur, removal would have caused considerable bone and soft tissue damage. Therefore, they used a Proxima short-stem, which is shorter compared to a conventional stem, but has a resection level similar to standard implants and also has a size which, at least at the proximal part, is as large as conventional stems.

In our patient's case, we used a metaphyseally anchored short-stem design, which clearly preserves more bone stock at the proximal femur but requires a resection level closely under the femoral head. By doing this, the femoral neck ring is preserved, which is needed for a firm anchorage of the implant. If those prerequisites are met, good primary stability of the SHA implant can be achieved. [14]

So far, good functional results and good shortterm and mid-term survival rates have been reported for various short-stem hip designs. [10-12] Advantages of SHA include a more physiologic load transfer at the metaphyseal part of the femur and reduced soft tissue trauma, as the small and curved designs facilitate the preparation of the femoral cavity and the insertion of the stem. [12] As a result, faster postoperative mobilization with a reduced hospital stay has been reported. [15]

A further advantage of SHA is the preservation of the femoral bone stock. This allows the use of a conventional stem should revision become necessary, thus avoiding revision implants with an inferior outcome. All acetabular cups, bearing surfaces, and head sizes that are used for conventional total hip arthroplasty can also be applied for SHA. For those reasons, SHA offers an attractive alternative 
for younger patients requiring hip replacement and, as shown in this report, can also be used to revise a HRA implant.

Regardless, it should be noted that to date, only short-term and mid-term results are available for SHA, and these results still have to be confirmed by long-term studies.

\section{Conclusion}

This case report demonstrates that revision of hip resurfacing arthroplasty can be performed not only with a conventional hip implant, but also with a bone-conserving short-stem hip implant. This is of particular importance, as it allows further preservation of the femoral bone stock and helps to provide a long-term solution to younger patients with a high risk of further revisions.

\section{Consent}

Written informed consent was obtained from the patient for publication of this case report and any accompanying images.

\section{Competing interests}

The authors declare that they have no competing interests.

\section{Author Information}

Florian Schmidutz, Lorenz Wanke-Jellinek, Volkmar Jansson, Andreas Fottner and Farhad Mazoochian are from the Department of Orthopedic Surgery, University of Munich (LMU), Campus Grosshadern, Munich, Germany.

\section{Source}

Schmidutz F, Wanke-Jellinek L, Jansson V, Fottner V, Mazoochian F. Revision of hip resurfacing arthroplasty with a bone-conserving short-stem implant. Journal of Medical Case Reports 2012, 6:249 doi:10.1186/1752-1947-6-249. http://www. jmedicalcasereports.com/content/6/1/249 (C) 2012 Schmidutz et al.; licensee BioMed Central Ltd. This is an Open Access article distributed under the terms of the Creative Commons Attribution License (http:// creativecommons.org/licenses/by/2.0), which permits unrestricted use, distribution, and reproduction in any medium, provided the original work is properly cited.

\section{References}

1. Swedish Hip Arthroplasty Register: Annual Report. http://www.shpr.se webcite

2. Lamberton TD, Kenny PJ, Whitehouse SL, Timperley AJ, Gie GA: Femoral impaction grafting in revision total hip arthroplasty: a follow-up of 540 hips. J Arthroplasty 2011, 26:1154-1160.

3. Pattyn C, Mulliez A, Verdonk R, Audenaert E: Revision hip arthroplasty using a cementless modular tapered stem. Int Orthop 2012, 36:35-41.

4. Ball ST, Le Duff MJ, Amstutz HC: Early results of conversion of a failed femoral component in hip resurfacing arthroplasty. J Bone Joint Surg Am 2007, 89:735-741.

5. Sandiford NA, Muirhead-Allwood SK, Skinner JA: Revision of failed hip resurfacing to total hip arthroplasty rapidly relieves pain and improves function in the early post operative period. J Orthop Surg Res 2010, 5:88.

6. Springer BD, Connelly SE, Odum SM, Fehring TK, Griffin WL, Mason JB, Masonis JL: Cementless femoral components in young patients: review and meta-analysis of total hip arthroplasty and hip resurfacing. $\mathrm{J} \mathrm{Ar}-$ throplasty 2009, 24:2-8.

7. Banerjee M, Bouillon B, Banerjee C, Bäthis H, Lefering R, Nardini M, Schmidt J: Sports activity after total hip resurfacing. Am J Sports Med 2010, 38:1229-1236

8. Australian Orthopaedic Association: Australian Orthopaedic Association National Joint Replacement Registry - Annual Report. 2010. https://aoanjrr.dmac.adelaide.edu.au/annual-reports-2010 webcite

9. Garrett SJ, Bolland BJ, Yates PJ, Gardner EM, Latham JM: Femoral revision in hip resurfacing compared with large-bearing metal-on-metal hip arthroplasty. J Arthroplasty 2011, 26:1214-1218.

10. Briem D, Schneider M, Bogner N, Botha N, Gebauer M, Gehrke T, Schwantes B: Mid-term results of 155 patients treated with a collum femoris preserving (CFP) short stem prosthesis. Int Orthop 2011, 35:655-660.

11. Schmidutz F, Grote S, Pietschmann M, Weber P, Mazoochian F, Fottner A, Jansson V: Sports activity after short-stem hip arthroplasty. Am J Sports Med 2012, 40:425-432.

12. Morrey BF, Adams RA, Kessler M: A conservative femoral replacement for total hip arthroplasty. A prospective study. J Bone Joint Surg Br 2000, 82:952-958.

13. Sanguesa-Nebot MJ, Soriano FC, Gabarda RF, Mordt CV: Revision hip arthroplasty with a short femoral component in fractured hydroxyapatite fully coated femoral stem. J Arthroplasty 2010, 25:1168-1176.

14. Fottner A, Schmid M, Birkenmaier C, Mazoochian F, Plitz W, Volkmar J: Biomechanical evaluation of two types of short-stemmed hip prostheses compared to the trust plate prosthesis by three-dimensional measurement of micromotions. Clin Biomech (Bristol, Avon ) 2009, 24:429-434.

15. Tahim AS, Stokes OM, Vedi V: The effect of femoral stem length on duration of hospital stay. Hip Int 2012, 22:56-61. 